\title{
Simple sequence repeat variation in the Daphnia pulex genome
}

\author{
Way Sung $^{1 *}$, Abraham Tucker ${ }^{1,3}$, R Daniel Bergeron², Michael Lynch ${ }^{3}$, W Kelley Thomas ${ }^{1}$
}

\begin{abstract}
Background: Simple sequence repeats (SSRs) are highly variable features of all genomes. Their rapid evolution makes them useful for tracing the evolutionary history of populations and investigating patterns of selection and mutation across gnomes. The recently sequenced Daphnia pulex genome provides us with a valuable data set to study the mode and tempo of SSR evolution, without the inherent biases that accompany marker selection.

Results: Here we catalogue SSR loci in the Daphnia pulex genome with repeated motif sizes of 1-100 nucleotides with a minimum of 3 perfect repeats. We then used whole genome shotgun reads to determine the average heterozygosity of each SSR type and the relationship that it has to repeat number, motif size, motif sequence, and distribution of SSR loci. We find that SSR heterozygosity is motif specific, and positively correlated with repeat number as well as motif size. For non-repeat unit polymorphisms, we identify a motif-dependent end-nucleotide polymorphism bias that may contribute to the patterns of abundance for specific homopolymers, dimers, and trimers. Our observations confirm the high frequency of multiple unit variation (multistep) at large microsatellite loci, and further show that the occurrence of multiple unit variation is dependent on both repeat number and motif size. Using the Daphnia pulex genetic map, we show a positive correlation between dimer and trimer frequency and recombination.

Conclusions: This genome-wide analysis of SSR variation in Daphnia pulex indicates that several aspects of SSR variation are motif dependent and suggests that a combination of unit length variation and end repeat biased base substitution contribute to the unique spectrum of SSR repeat loci.
\end{abstract}

\section{Background}

Tandem arrays of DNA nucleotides, known as simple sequence repeats (SSR), are extremely dynamic parts of the genome. These tandem repeats vary in motif sequence, length, and repeat number. The most common SSR loci are homopolymers (repeated single nucleotide), dimers (repeated nucleotide pair), and trimers (repeated nucleotide triplet). The highly polymorphic nature of SSRs makes them desirable for use in both genotyping and population-level evolutionary studies. Simple sequence repeats may influence the fitness of the organism [1], and in specific cases are known to be causal of human disease [1].

A high mutation rate at SSRs has been well documented in a number of organisms using microsatellite

\footnotetext{
* Correspondence: wsung@cisunix.unh.edu

'Hubbard Center for Genome Studies, University of New Hampshire,

Durham, NH 03824, USA

Full list of author information is available at the end of the article
}

constructs, pedigree analyses, and mutation accumulation (MA) experiments [2-4]. The high mutation rate in SSRs is due to a propensity for DNA misalignment during replication [5], regulated primarily by the universal mismatch repair system (MMR). MMR knockout experiments show dramatic increases (up to 100 fold greater) in the rate of simple sequence variation [6,7]; and suggest that surveillance by MMR may vary across the genome [8]. Although the MMR system has been wellcategorized in certain species, the components of MMR may vary from species to species, which may result in lineage specific patterns of SSR repair, and consequently lineage specific patterns of SSR variation [9]. In addition to MMR fidelity; repeat number, motif sequence, motif size, local rates of recombination and genomic location can influence rates of SSR variation $[8,10,11]$. Genome wide analysis of SSR variation in well-characterized systems will facilitate a greater understanding of the
C Biomed Central

() 2010 Sung et al; licensee BioMed Central Ltd. This is an Open Access article distributed under the terms of the Creative Commons Attribution License (<url>http://creativecommons.org/licenses/by/2.0</url>), which permits unrestricted use, distribution, and reproduction in any medium, provided the original work is properly cited. 
relationship between MMR evolution, and the abundance of SSR and levels of variation at these loci.

Daphnia pulex (water flea), crustacean represents a particularly useful platform for the study of SSR evolution. The Daphnia pulex lineage is distinct from the two model organisms Caenorhabditis elegans (nematode) and Drosophila melanogaster (fruit fly), both of which have well characterized simple sequence repeat variation $[2,12]$. The genome sequence of $D$. pulex is based on a single heterozygous genotype sampled from a natural population with minimal inbreeding [13]. The heterozygosity within the genome sequence can be assayed by analysis of the raw sequence reads $(8 \times$ coverage) allowing a nearly genome-wide analysis of the variation at SSR loci.

There are two main goals of this study. The first is to provide a detailed catalog of SSR loci and their distribution within the genome of Daphnia pulex. The second goal is to assay SSR heterozygosity on a genome wide scale to test for motif specific rates and patterns of SSR evolution.

\section{Results and Discussion}

\section{Catalog and Distribution of SSR loci}

In order to enumerate all types of SSRs in the Daphnia pulex genome, we first identified all SSR loci in the largest 100 scaffolds (Daphnia pulex assembly 9/01/2006; $\mathrm{N} 50=103)$ with repeat motifs from 1 to 100 nucleotides, repeated perfectly three or more times. Motif size is defined by the length of the set of nucleotides that are repeated, while repeat number reflects the number of times that set is repeated. For example, the nucleotides ATATAT have a motif size of 2 (AT) and the number of repeats is 3 . Under these criteria, we identified a total of 7,229,342 perfect SSRs, spanning 48.4 Mbp (21.3\%) of the Daphnia pulex genome (Figure 1). As with all prior studies, the abundance of all SSR types exceeds random expectations based on nucleotide composition [14]. Homopolymeric repeats (HPs) make up 93\% of all SSRs (6726771 loci), followed distantly by dimers $(4.8 \% ; 347288$ loci) and trimers $(1.8 \% ; 133428$ loci). The remaining SSRs with motifs larger than 3 base pairs constitute a much smaller fraction of the genome. The distribution of SSR in the Daphnia pulex genome most closely resembles the SSR distribution in Caenorhabditis elegans (93\% HP, 5.3\% dimers, 1.3\% trimers) (Figure 2).

The number of simple sequence repeats follows an exponential decay with increasing motif size (Figure 1). The motifs with the size class of 24, 36, 42, 54, and 60 deviating from the regression line (Red in Figure 1). Closer analysis of these motif classes using $D$. pulex genome annotations [15] reveals that the overrepresented motifs are protein coding sequences of tandemly

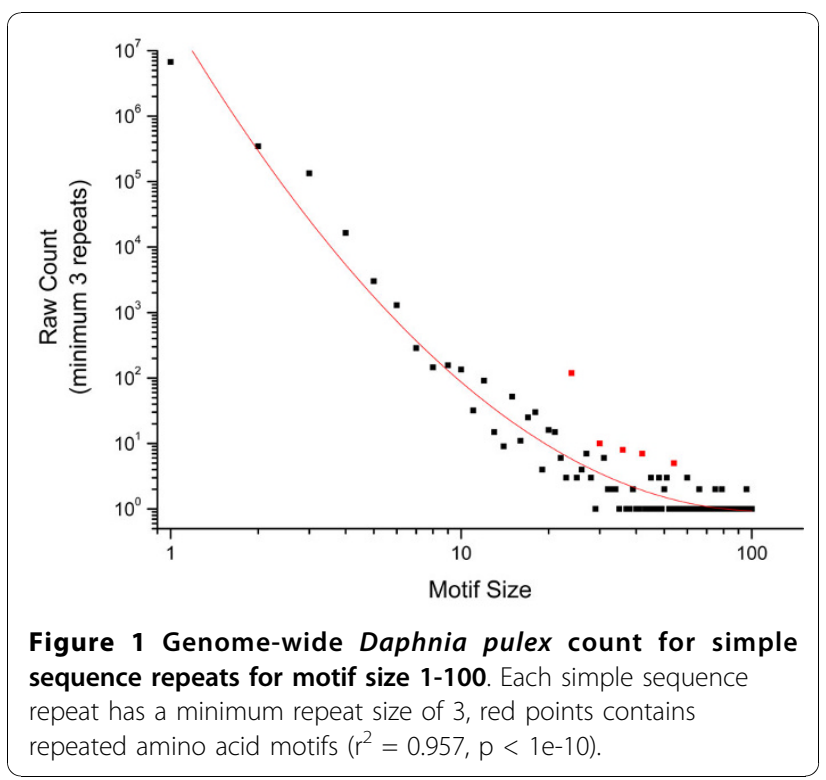

repeated amino acid motifs found in multiple copies that are spread across the genome. For example, the 24 mers are multiple copies of an 8 amino acid repeat found in the largest subunit of DNA-directed RNA polymerase II. This inflates the count of these motifs, all of which are necessarily divisible by one codon unit.

\section{Abundance of Specific SSR Motif Types}

The number of $\mathrm{A} / \mathrm{T}$ homopolymers $(5,276,283)$ vastly exceeds the number of $\mathrm{G} / \mathrm{C}$ homopolymers $(1,450,489)$. The ratio of $\mathrm{A} / \mathrm{T}$ to $\mathrm{G} / \mathrm{C}$ homopolymers is not predicted by the Daphnia pulex base composition (A/T 59\%, G/C $41 \%, \mathrm{p}=0)$. The overabundance of $\mathrm{A} / \mathrm{T}$ homopolymer

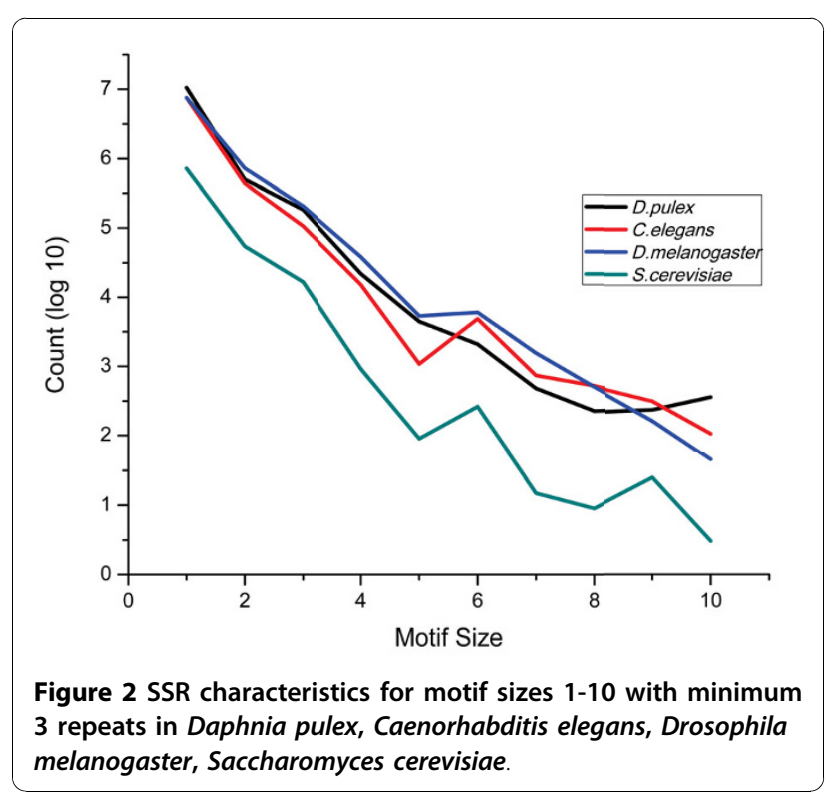


tracts may reflect unique origins of the $\mathrm{A} / \mathrm{T}$ homopolymers from A/T rich transposable elements [16,17] or base specific mutational patterns in repetitive sequence motifs [14,17].

When studying SSR loci, genome-wide annotations commonly combine repeat motifs into complementary and overlapping DNA pairings [4], such that $\mathrm{CA}=\mathrm{AC}=$ $\mathrm{GT}=\mathrm{TG}, \mathrm{GA}=\mathrm{AG}=\mathrm{CT}=\mathrm{TC}, \mathrm{AT}=\mathrm{TA}$, and $\mathrm{GC}=$ CG. In each case, the first two motifs of each set (e.g. CA/ $\mathrm{AC}$ ) represent cases where the longest perfect repeat begins with each of the two bases and the second pair of motifs (e.g. GT/TG) are the complementary base. Our approach counts each of the 12 dimer motifs independently and determines the maximum size of a perfect microsatellite repeat regardless of the starting base. Using these criteria, we observe a bias in the starting (and ending) nucleotide of dimer repeat loci. While the number of repeats from each of the two strands is necessarily equivalent, the starting base for each motif is significantly biased in all cases for both dimers and trimers (Table 1). For trimers, we examined the overall codon usage for Daphnia pulex, and did not see any correlation between motif class representation and codon usage (Table 1 ).

One explanation for the unexpected count differential between motifs in a similar grouping is the existence of a motif specific pattern of end base substitution, a phenomenon that has been previously observed in chicken microsatellites [18]. These observations may be informative with regard to the origin, or maintenance of SSR loci. To explore these patterns further, we analyzed variation at all SSR loci within the D. pulex genome assembly involving repeat number, incomplete insertion deletion events (indels), and base substitutions.

\section{SSR heterozygosity}

Multiple models have been developed to explain the pattern of SSRvariation, such as the stepwise mutation model (SMM) [19], and more recent models that focus on a balance between the rate and pattern of length variation and base substitutions [20]. To evaluate the relative levels of these two processes, we analyzed the number of loci that were heterozygous for motif unitlength variation and non-repeat unit polymorphism (NRUPs). First, we identified all SSR loci that showed significant evidence of repeat length variation in the raw sequence data from which the genome was assembled (see methods). In Daphnia pulex, we were able to assay $6,062,268$ of the 7,229,342 total SSRs for heterozygosity, of which 23,360 of the SSRs varied in length by at least one perfect repeat. Although smaller scaffolds may contain additional repeats, there is a increased possibility that these smaller scaffolds are either contaminated sequences or paralogous sequences, therefore we excluded them from the heterozygosity analysis.
For the five most abundant SSR motif sizes (1-5bp), we observe either an exponential or linear increase in repeat length heterozygosity as repeat number increases (Figure 3). Repeats with motif size greater than 5 were analyzed, however, due to the limited sample of large motifs, we were unable to discern any distinguishable pattern. The pattern observed in D. pulex for motifs with size 1-5bp is consistent with the previous observation in yeast and humans that mutation rate increases are correlated with increasing repeat number [7,17,21-23]. The observed pattern is unlikely to reflect SSR sequencing errors because while the frequency of sequencing errors does increase with the number of repeats, it is unlikely that the error will result in the variation of a perfect repeat unit.

We then further dissect repeat length heterozygosity, focusing on potential differences in heterozygosity among specific motif types. Due to the lack of heterozygotes in motifs longer than dimers, this analysis is limited to the two homopolymer and four dimer repeat motifs (Figure 4a and 4b). Homopolymers in Daphnia pulex exhibit increased repeat heterozygosity with increasing repeat number reaching a maximal rate of increase between 6 and 12 repeats $\left(A / \mathrm{T} \mathrm{r}^{2}=0.925, \mathrm{G} / \mathrm{C}\right.$ $\left.\mathrm{r}^{2}=0.899\right)$. The $\mathrm{G} / \mathrm{C}$ motif achieves a much higher frequency of heterozygosity (>20\%) than A/T loci ( 10\%). The higher level of heterozygosity in $\mathrm{G} / \mathrm{C}$ loci is consistent with direct estimates of the mutation rate using Caenorhabditis elegans mutation accumulation lines, where the $\mathrm{G} / \mathrm{C}$ homopolymer mutation rate was $\sim 20$ fold greater than that for A/T HP loci [6], and consistent with previous experiments on $E$. coli, human, and yeast HPs which show a higher mutation rate of $\mathrm{G} / \mathrm{C}$ HPs attributed to possible differences in base stacking properties during replication [24]. While it is possible that $\mathrm{G} / \mathrm{C}$ loci with large numbers of repeats are being selectively excluded due to higher sequencing error rates, the fact that the $\mathrm{A} / \mathrm{T}$ loci with lower levels of heterozygosity also show this plateau suggests that it may not simply be a sampling bias. If we assume that replication errors increase proportionately with an increase in repeat number, a plateau of $\mathrm{A} / \mathrm{T}$ and $\mathrm{G} / \mathrm{C}$ loci suggest that there may be a length threshold for differential repair activity, length-dependent counter mutation, or selection.

Similar to homopolymer repeats, dimer repeat heterozygosity increases with increasing repeat number $\left(\mathrm{AC} \mathrm{r}^{2}\right.$ $=0.862 ; \mathrm{AG}^{2}=0.891, \mathrm{AT}^{2}=0.736, \mathrm{CG} \mathrm{r}^{2}=0.539$ ) (Figure 4d). Our analysis indicates that the motifs AC and AG show the highest rate of increase in heterozygosity $(1.22 \%$ and $0.99 \%$ per repeat unit) followed by AT and CG motifs $(\sim 0.75 \%$ per increase in repeat unit). In a pedigree study of forty-two microsatellite loci using six different Drosophila melanogaster populations, AC/AG 
Table 1 Abundance and Starting Nucleotide Preference for homopolymer and dimer loci in D. pulex

\begin{tabular}{|c|c|c|c|c|c|c|c|c|c|}
\hline \multicolumn{10}{|l|}{ Dimers } \\
\hline Motif Type & Count (obs) & End (exp) & p-value & Starting Pref. & Motif Type & Count (obs) & End (exp) & p-value & Starting Pref. \\
\hline TA & 48814 & 42186 & 0 & $\mathrm{~T}$ & GC & 8444 & 7623 & $1 \mathrm{E}-39$ & G \\
\hline AT & 35558 & 42186 & & & CG & 6802 & 7623 & & \\
\hline GA & 33951 & 31919 & 0 & $\mathrm{G} / \mathrm{T}$ & $A C$ & 33773 & 29999 & 0 & $A / T$ \\
\hline$A G$ & 30185 & 31919 & & & CA & 26535 & 29999 & & \\
\hline $\mathrm{TC}$ & 40029 & 31919 & & & $\mathrm{TG}$ & 35249 & 29999 & & \\
\hline CT & 23511 & 31919 & & & GT & 24437 & 29999 & & \\
\hline \multicolumn{10}{|l|}{ Trimers } \\
\hline $\mathrm{AAC}+$ & 2728 & 2821 & $1 \mathrm{E}-78$ & $\mathrm{~T} / \mathrm{C}$ & $\mathrm{ACT}$ & 598 & 695 & $7 E-15$ & $\mathrm{G} / \mathrm{T}$ \\
\hline $\mathrm{ACA}^{*}$ & 2431 & 2821 & & & CTA+ & 734 & 695 & & \\
\hline$C A A^{*}$ & 3380 & 2821 & & & TAC+ & 791 & 695 & & \\
\hline $\mathrm{GTT*}$ & 2339 & 2821 & & & AGT & 665 & 695 & & \\
\hline $\mathrm{TGT}^{*}$ & 2657 & 2821 & & & TAG+ & 564 & 695 & & \\
\hline$\pi T G^{*}$ & 3390 & 2821 & & & GTA+ & 815 & 695 & & \\
\hline AAG & 5734 & 4486 & 0 & $\mathrm{~T} / \mathrm{A}$ & AGC+ & 1839 & 2823 & $1 \mathrm{E}-226$ & $C / G$ \\
\hline$A G A^{*}$ & 3657 & 4486 & & & $G C A^{*}$ & 2363 & 2823 & & \\
\hline $\mathrm{GAA}^{*}$ & 4278 & 4486 & & & CAG+ & 4131 & 2823 & & \\
\hline $\mathrm{CTT}$ & 3393 & 4486 & & & $\mathrm{GCT}$ & 3115 & 2823 & & \\
\hline TCT & 3692 & 4486 & & & TGC+ & 2725 & 2823 & & \\
\hline TTC+ & 6161 & 4486 & & & $\mathrm{CTG}$ & 2767 & 2823 & & \\
\hline AAT* $^{*}$ & 4099 & 2937 & $5 E-216$ & A & AGG+ & 971 & 1039 & $2 \mathrm{E}-18$ & $\mathrm{G} / \mathrm{T}$ \\
\hline ATA+ & 2260 & 2937 & & & $\mathrm{GGA}^{*}$ & 1222 & 1039 & & \\
\hline $\mathrm{TAA}^{*}$ & 2406 & 2937 & & & GAG+ & 988 & 1039 & & \\
\hline ATT* & 3533 & 2937 & & & $\mathrm{CCT}$ & 905 & 1039 & & \\
\hline TAT* & 2233 & 2937 & & & TCC & 1207 & 1039 & & \\
\hline TTA & 3093 & 2937 & & & CTC & 940 & 1039 & & \\
\hline $\mathrm{ACC}+$ & 855 & 1089 & $4 \mathrm{E}-40$ & $\mathrm{C} / \mathrm{T}$ & ATC & 1153 & 1404 & $8 \mathrm{E}-46$ & $\mathrm{~T}$ \\
\hline CAC+ & 1057 & 1089 & & & $\mathrm{TCA}^{*}$ & 1703 & 1404 & & \\
\hline $\mathrm{CCA}^{*}$ & 1383 & 1089 & & & CAT $^{*}$ & 1342 & 1404 & & \\
\hline GGT & 1034 & 1089 & & & $\mathrm{GAT}^{*}$ & 1452 & 1404 & & \\
\hline GTG & 921 & 1089 & & & TGA & 1661 & 1404 & & \\
\hline TGG* & 1285 & 1089 & & & ATG* $^{*}$ & 1111 & 1404 & & \\
\hline ACG & 1229 & 1373 & $8 \mathrm{E}-11$ & G & CCG & 703 & 742 & $1 \mathrm{E}-47$ & G \\
\hline CGA & 1406 & 1373 & & & CGC+ & 519 & 742 & & \\
\hline GAC+ & 1561 & 1373 & & & GGC & 916 & 742 & & \\
\hline CGT & 1325 & 1373 & & & CGG & 731 & 742 & & \\
\hline TCG & 1267 & 1373 & & & GCG+ & 585 & 742 & & \\
\hline GTC & 1452 & 1373 & & & GCC & 995 & 742 & & \\
\hline
\end{tabular}

This table shows nonrandom starting nucleotides in both dimers and trimers. Motif type indicates largest possible repeat identified for each staggered SSR set. Pvalue calculated using Pearson's chi-square test for random expectation based upon observed and expected frequencies.

Preferential starting base is determined by the highest frequency SSR for the motif grouping. ${ }^{*}$ indicate highly used codons, + indicate rarely used codons. 


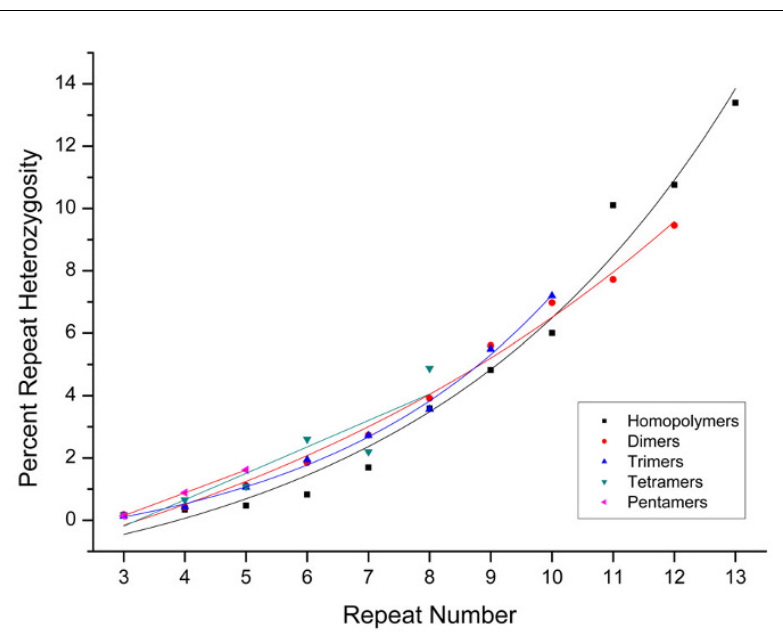

Figure 3 Percent repeat heterozygosity for each motif size in Daphnia pulex. The $X$-axis is the repeat number found in the assembly. The Y-axis shows the percent of loci at that motif size that are heterozygous. Motif sizes best fit 1-3 an exponential curve while motif sizes 4 and 5 best fit a linear pattern. (Motifs 1-5 respectively: $r^{2}=0.975, p<1 e-7 ; r^{2}=0.990, p<1 e-8 ; r^{2}=0.995, p$ $\left.<1 e-6 ; r^{2}=0.824, p<.01 ; r^{2}=0.999, p<.01\right)$.

repeats were shown to vary in repeat size at $\sim 3$-fold and 1.5-fold more than AT repeats, with GC repeats not surveyed [12]. All other things being equal, both Daphnia pulex and Drosophila melanogaster have similar patterns of repeat variation such that the order of variation by motif sequence is $\mathrm{AC} / \mathrm{AG}>\mathrm{AT}>\mathrm{GC}$.

Studies in yeast and C. elegans have shown that the homopolymer mutation rate is $\sim 100$-fold greater than the mutation rate at dimer loci [3,7]. On a per locus basis, the percent heterozygosity of homopolymer repeats and dimer repeats are nearly identical in Daphnia pulex (+/- 0.5\%), with the largest differences occurring at large repeats. Together, these observations suggest that the homopolymer and dimer mutation rates may be more similar in the Daphnia genome or that selection severely limits variation at homopolymer loci.

\section{Non-repeat Unit Polymorphisms}

Non-repeat unit polymorphisms (NRUPs) are base substitutions or indels that interrupt the continuity of SSR locus and can shape the observed abundance of each repeat class (eg. CACATCACA is an interruption of a CA dimer with a $\mathrm{T}$ ). It is extremely complicated to define and compare imperfect repeats, so for our analysis we identified SSR loci that are perfect repeats in the assembly, and subsequently looked for the existence of NRUPs in the reads aligned to each locus.

Examination of the NRUPs at HP loci (Figure 4c) reveals that as repeat number increases, the proportion of polymorphisms per nucleotide increases $\left(\mathrm{C} / \mathrm{G} \mathrm{r}^{2}=\right.$ $\left.0.905, \mathrm{p}<1 \mathrm{e}-12 ; \mathrm{A} / \mathrm{T}^{2}=0.446, \mathrm{p}<.01\right)$. The rate of increase is particularly pronounced between 6 and 12 bases at HPs. We further evaluated the positions of NRUPs in a locus by comparing the site of polymorphism to the random expectation (Table 2). The result shows that at HP loci, point mutations occur at the beginning or end nucleotides at a higher frequency than expected, and the proportion increases with increasing repeat number, effectively shortening or lengthening the HP loci by a single unit. At smaller and more abundant repeat numbers (from 3-12 nucleotides), the number of end-point polymorphisms is substantial when compared to length variation, and point mutations may have a significant influence on homopolymer equilibrium.

Similar to homopolymers, a significant end bias is found for NRUPs within dimer repeats (Table 2). At dimer loci with few repeats (less than 5), NRUPs outnumber unit length indels, while at loci with 6 or more repeats, unit length indels contribute to the majority of variation (Table 2). This pattern matches results found in a microsatellite study of the chicken genome, which also shows an end bias NRUP distribution within repeat arrays [18]. The propensity for end nucleotide polymorphisms at short repeats can lead to a SSR distribution characterized by species-specific mutation biases. Our analysis does not allow us to distinguish which of the heterozygous alleles is ancestral or derived and it would be interesting to understand if the rate of single nucleotide gains and losses are equivalent. A genomewide SSR study involving two closely related populations with a recent out group would be required to determine how NRUPs effect the equilibrium of SSR length distribution in an organism.

For at least chicken and D. pulex, these observations suggest that the specific abundance of specific types of microsatellites within a genome reflects a dynamic balance between changes in repeat number and base substitution. When combined with unequal patterns of base substitution, these observations can explain the overabundance of specific starting and ending nucleotides in SSR loci (Table 1).

\section{Multi-step variation}

Recent human microsatellite studies suggest that multistep variation is a significant component of variation at larger SSR loci [25], deviating from the SSM model. Furthermore, researchers have shown that the directionality of multi-step mutations is length-dependent with a critical repeat number at which contractions are more frequent than expansions [26], while others suggest that a critical number does not exist [27]. In this experiment, we required that each multistep variant displayed a minimum of at least two full perfect repeats. Our analysis revealed two patterns. First, for all motif sizes, the 


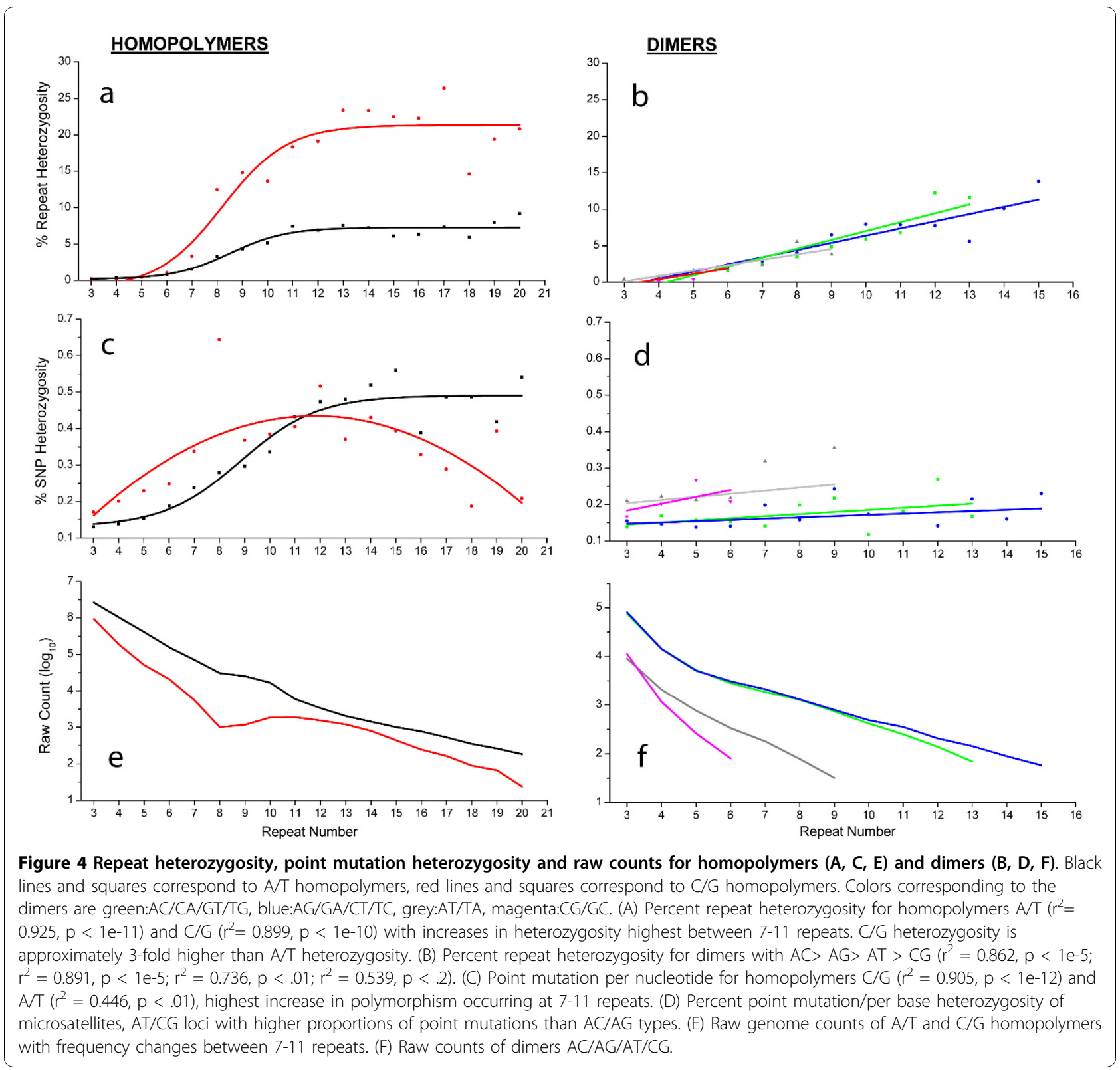

proportion of heterozygotes that are multi-step increases with the number of repeats at a locus (Figure 5). Second, the rate of multi-step variation increases with increased motif size. Further analysis of individual homopolymer motif types showed that the fraction of multistep variation at A/T HP is much lower $(12.14 \%$ or 2129/17544) than that of G/C HPs (27.4\% or 972/3547) (Figure 5). Dimer loci also show a high frequency ( $21.3 \%$ or $288 / 1353)$ of multistep polymorphisms, with AC motif showing the highest proportion multistep increase (0.0423) followed by AG (0.036) and AT (0.0301) (Figure 5). Direct estimates of dimer SSR loci in D. pulex and C. elegans show that $73 \%$ (173 of 237) of the variation at AC and AG loci larger than 13 repeats was multi-step variants [26]. A similar partition of our data reveals that $52 \%(12 / 23)$ of AC and AG dimer loci with greater than 13 repeats are multi-step differences. Although the experiments described above show that most of these multi-step changes are repeat length increases, our method of analysis does not allow us to polarize the differences between alleles. In any event, there appears to be a motif dependant correlation between repeat length and multi-step mutation change for SSR loci.

\section{Density of SSR Loci}

Although the factors that affect the density of SSR loci within a genome remain to be clearly defined, the 
Table 2 Position of the interruption in imperfect SSR.

\begin{tabular}{|c|c|c|c|c|c|}
\hline \multirow[b]{2}{*}{$\begin{array}{l}\text { Repeat } \\
\text { Size }\end{array}$} & \multicolumn{5}{|c|}{ Homopolymers } \\
\hline & $\begin{array}{l}\text { End } \\
\text { (obs) }\end{array}$ & $\begin{array}{l}\text { Middle } \\
\text { (obs) }\end{array}$ & $\begin{array}{l}\text { End } \\
\text { (exp) }\end{array}$ & $\begin{array}{c}\text { Middle } \\
\text { (exp) }\end{array}$ & $\begin{array}{c}\mathrm{p}- \\
\text { value }\end{array}$ \\
\hline 3 & 15,005 & 50 & 10,037 & 5,018 & 0 \\
\hline 4 & 5,793 & 1,392 & 3,593 & 3,593 & 0 \\
\hline 5 & 2,549 & 1,168 & 1,487 & 2,230 & 5.E-277 \\
\hline 6 & 1,309 & 735 & 681 & 1,363 & 1.E-190 \\
\hline 7 & 810 & 492 & 372 & 930 & 5.E-159 \\
\hline 8 & 447 & 294 & 185 & 556 & 3.E-109 \\
\hline 9 & 459 & 253 & 158 & 554 & 7.E-162 \\
\hline 10 & 350 & 285 & 127 & 508 & 2.E-108 \\
\hline 11 & 178 & 191 & 67 & 302 & 1.E-50 \\
\hline 12 & 119 & 167 & 48 & 238 & 1.E-29 \\
\hline 13 & 65 & 121 & 29 & 157 & 1.E-13 \\
\hline 14 & 45 & 107 & 22 & 130 & 7.E-08 \\
\hline 15 & 34 & 77 & 15 & 96 & 8.E-08 \\
\hline 16 & 18 & 43 & 8 & 53 & 6.E-05 \\
\hline 17 & 12 & 40 & 6 & 46 & 1.E-02 \\
\hline 18 & 6 & 28 & 4 & 30 & 2.E-01 \\
\hline 19 & 3 & 23 & 3 & 23 & 9.E-01 \\
\hline \multirow[t]{2}{*}{20} & 6 & 15 & 2 & 19 & 5.E-03 \\
\hline & \multicolumn{5}{|c|}{ Dimers } \\
\hline Repeat Size & End (obs) & Middle (obs) & End (exp) & Middle (exp) & p-value \\
\hline 3 & 1,588 & 641 & 1,486 & 743 & 5.E-06 \\
\hline 4 & 332 & 208 & 270 & 270 & 9.E-08 \\
\hline 5 & 121 & 83 & 82 & 122 & 2.E-08 \\
\hline 6 & 65 & 61 & 42 & 84 & 1.E-05 \\
\hline 7 & 58 & 53 & 32 & 79 & 3.E-08 \\
\hline 8 & 47 & 29 & 19 & 57 & 1.E-13 \\
\hline 9 & 39 & 30 & 15 & 54 & 7.E-12 \\
\hline 10 & 17 & 10 & 5 & 22 & 2.E-08 \\
\hline 11 & 11 & 7 & 3 & 15 & 2.E-06 \\
\hline 12 & 9 & 7 & 3 & 13 & 2.E-05 \\
\hline 13 & 7 & 4 & 2 & 9 & 9.E-06 \\
\hline
\end{tabular}

This table shows the position of interruptions in simple sequence repeats. "End" indicates polymorphism at beginning or end of SSR loci, while middle variation indicates polymorphism occurs at interior positions (eg. Homopolymer with repeat length of three has two possible end nucleotide positions and one possible middle position. P-value is determined using Pearson's chi-square test for random expectation.

density of SSRs within genomes has been positively correlated with regional rates of recombination in Drosophila melanogaster [27], Saccharomyces cerevisiae [28], and C. elegans [2]. To evaluate the density of SSR loci in the Daphnia genome, we ranked the density of SSRs in each of the top 100 scaffolds ranging in size from $4.19 \mathrm{Mb}$ to $0.47 \mathrm{Mb}$ (Table 3a). Several scaffolds (68, 81, $83,89)$ show a dearth of HP loci and scaffolds 66 and 98 have a paucity of SSRs with five and six base motifs respectively. While no scaffolds showed an overabundance of homopolymers, several scaffolds showed significantly high numbers of SSRs, in particular scaffolds 43,

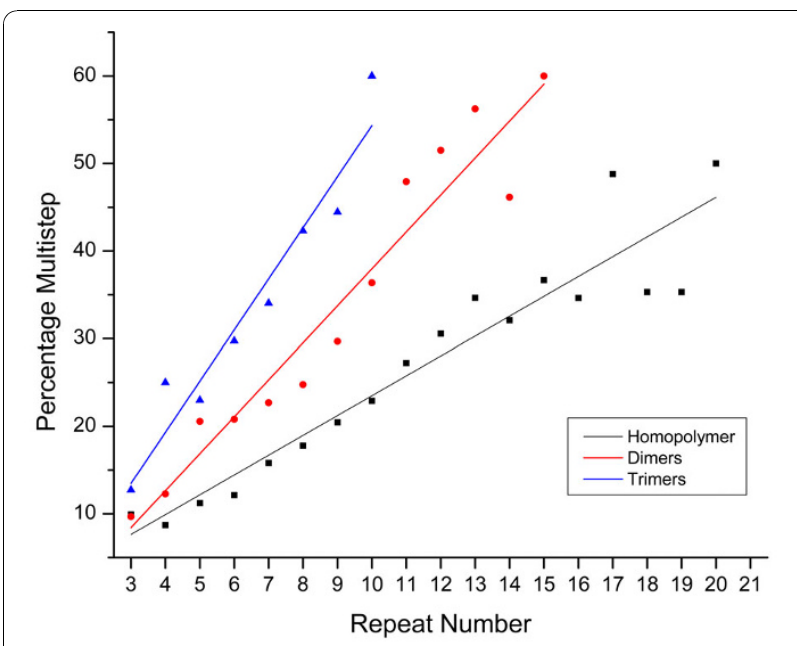

Figure 5 Percentage of repeat heterozygosity that involves more than a single unit. Homopolymers $r^{2}=0.896, p<1 e-8$; Dimers $r^{2}=0.927, p<1 e-7$; Trimers $r^{2}=0.927, p<1 e-4$.

74 , and 76, which have an overabundance of repeats of motif sizes greater than $1 \mathrm{bp}$. Simple sequence repeats have been shown to play a role in gene regulation [1] and consequently would be in close proximity to genes. To test if these patterns are correlated with gene density, we compared SSR scaffold abundance to Daphnia gene predictions [15], and show that none of the overabundant scaffolds listed previously are either gene poor or gene rich (Table 3a). Results found in C. elegans [6] also show little correlation between gene density and SSR density.

We then used a preliminary genetic map for Daphnia pulex [29] to test for correlations between SSR density and heterozygosity with rates of recombination within scaffolds. Based on 61 intervals in the genetic map that could be assigned to physical intervals in the top 100 scaffolds, we find that the density of microsatellite loci with motif sizes greater than $1 \mathrm{bp}$ show a significant positive correlation with the rate of recombination (Figure 6). This result is consistent with a yeast study showing a high frequency of microsatellite repeats near meiotic hotspots [28], and further supports role for SSR loci in the regulation of recombination. Both microsatellite repeats (repeats of motif size 2-6), and homopolymer repeats (single nucleotide repeats) correlate positively with recombination rate (Figure 7). Homopolymers have array sizes that are considerably larger than microsatellites, giving them properties that may influence recombination frequency.

Within the same intervals, no correlation was observed between microsatellite heterozygosity and recombination in Daphnia pulex (Figure 8). A previous microsatellite survey in humans [30] also showed no significant correlation between microsatellite heterozygosity 
Table 3 Gene content vs SSR abundance, and G/C content vs SSR heterozygosity

\begin{tabular}{|c|c|c|c|c|c|c|c|c|}
\hline \multicolumn{9}{|c|}{ Table 3a: Gene content vs SSR abundance } \\
\hline \multicolumn{9}{|c|}{ Motif Size } \\
\hline Abundance & 1 & 2 & 3 & 4 & 5 & 6 & 2 to 6 & Gene Content \\
\hline scaffold_18 & & & & & & & & + \\
\hline scaffold_32 & & + & & & + & & + & \\
\hline scaffold_43 & & + & & & & & + & \\
\hline scaffold_58 & & & & & + & & & \\
\hline scaffold_59 & & & & & & + & & \\
\hline scaffold_62 & & & & & + & & & \\
\hline scaffold_66 & & & & & - & & & \\
\hline scaffold_67 & & & & & & + & & + \\
\hline scaffold_68 & + & & & & & & & \\
\hline scaffold_74 & & + & & & & & + & - \\
\hline scaffold_76 & & + & & + & & + & & \\
\hline scaffold_81 & + & & & & & & & \\
\hline scaffold_82 & + & & & & + & & & - \\
\hline scaffold_84 & & & & & & & & - \\
\hline scaffold_85 & & & & & & + & & \\
\hline scaffold_88 & & & & & + & & & \\
\hline scaffold_90 & & & + & + & & & & \\
\hline scaffold_98 & & & & & & - & & \\
\hline
\end{tabular}

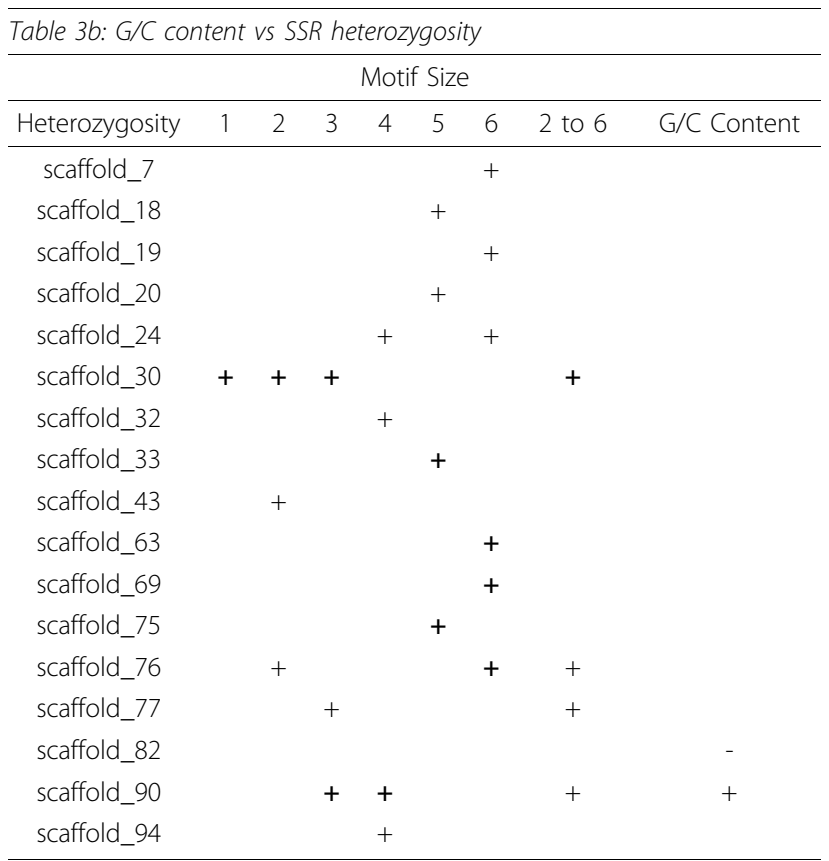

This table shows motif sizes that are significantly greater than (+) or less than $(-)$ the overall scaffold mean.

Motif Sizes that are significantly different (2 standard deviations) from the overall scaffold mean using Generalized Extreme Studentized Deviate (ESD) are bolded ( $p<0.05, d f=98$ ). Column 2 to 6 is the cumulative sum of motif sizes 2 to 6 (homopolymers excluded).

and recombination rate, however, the scale of the recombination intervals and their measurement often make such observations difficult. Although SSRs of all

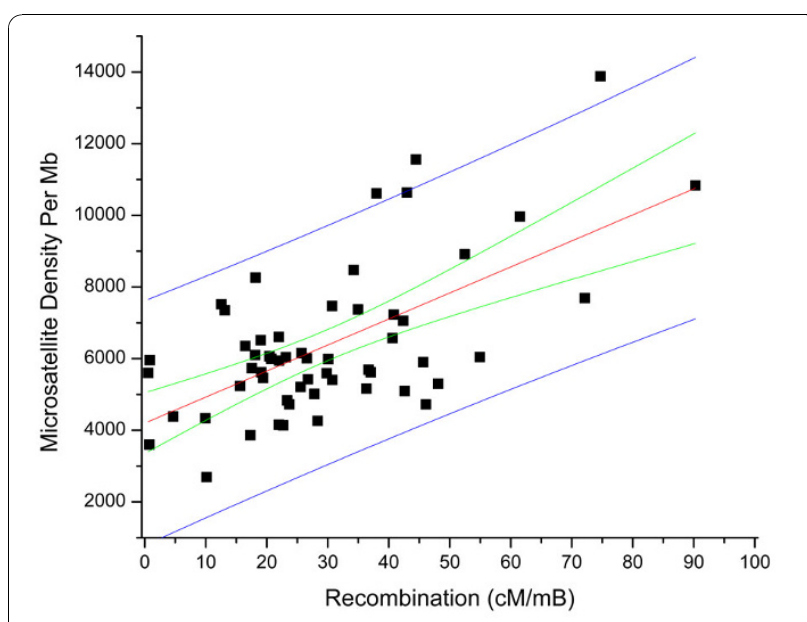

Figure 6 Positive correlation between recombination (cM/mB) and microsatellite density per $\mathbf{M b}$. 95\% prediction interval represent the blue line, $95 \%$ confidence interval represent the green line $\left(r^{2}=0.376, p<1 e-6\right)$.

types are correlated with recombination rates, the level of SSR heterozygosity seems uninfluenced by recombination.

The distribution of heterozygosity at SSR loci can be shaped by selective sweeps, background selection and locus-specific differences in mutation rates. Many of these patterns may themselves be correlated. Based on our analysis, scaffolds 24, 30, 76, and 90 have multiple motif classes that are significantly more heterozygous than expected (Table 3b). Scaffold 90 also shows significantly higher $\mathrm{G} / \mathrm{C}$ content (44\%; average $\mathrm{G} / \mathrm{C}=41 \%$ ), which is contradictory to suggestions of an evolved efficiency of MMR in G/C rich regions of eukaryotes [31].

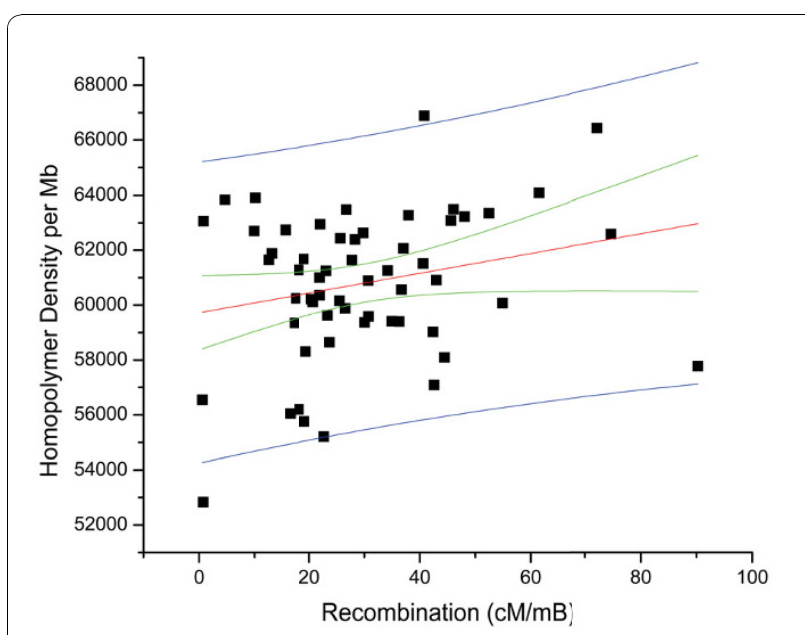

Figure 7 Lack of correlation between recombination $(\mathrm{cM} / \mathrm{mB})$ and microsatellite heterozygosity. $95 \%$ prediction interval represent the blue line, 95\% confidence interval represent the green line $\left(r^{2}=0.030, p>.1\right)$. 


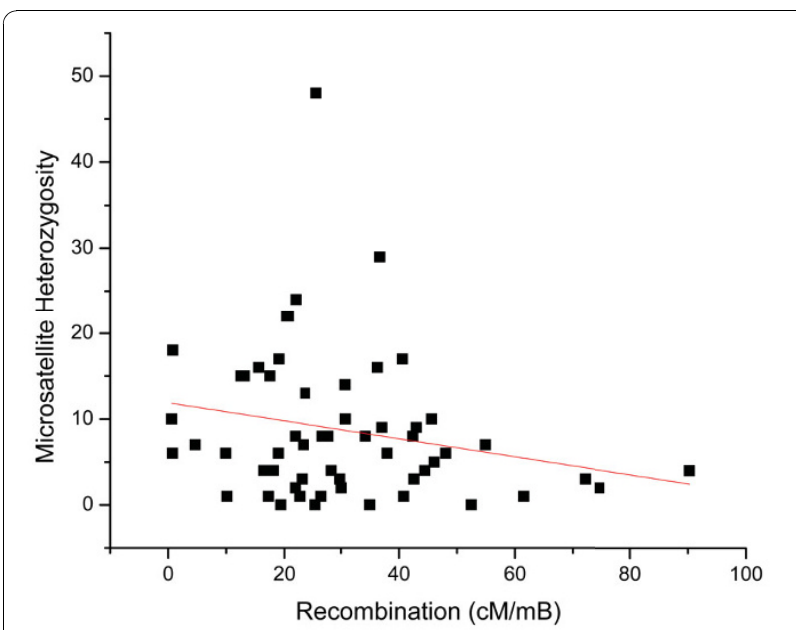

Figure 8 Positive correlation between recombination (cM/mB) and microsatellite density per $\mathbf{M b}$. $95 \%$ prediction interval represent the blue line, 95\% confidence interval represent the green line $\left(r^{2}=0.401, p<0.08\right)$

\section{Conclusions}

The Daphnia pulex genome sequence provides us with a unique opportunity to study genome-wide SSR patterns using whole genome shotgun reads. Although recent sequencing technologies have greatly improved sequencing throughput, these new technologies introduce multiple errors that are absent from WGS sequencing. Furthermore, the large read size of WGS sequencing allows for alignment to larger motif types that smaller read lengths cannot span. Daphnia pulex has undergone a minimal amount of inbreeding prior to sequencing, retaining high relative levels of assayable SSR heterozygosity that may be removed from heavily inbred genome sequencing projects.

SSRs, primarily microsatellites, are used commonly as genetic markers for population level studies. Our results show that in Daphnia pulex, levels of genome-wide SSR heterozygosity can not only vary with the length of motifs, but also by motif type in agreement with the studies of other organisms $[18,22,27]$. Our genome-wide results show that microsatellite loci with less than 6 repeats accumulate non-repeat unit polymorphisms at a greater rate than repeat length differences. In addition, AC/AG microsatellites accumulate repeat length differences at a greater rate than AT/GC microsatellites. Taken together, for Daphnia pulex, AC/AG repeats with a minimum of 6 repeats will provide the most resolution when used as genetic markers in Daphnia specific population level studies. Although the two available genomewide microsatellite studies of fruit-fly [27] and chicken [18] display similar patterns of heterozygosity (highest AC/AG heterozygosity), microsatellites studies from additional taxa must be made before a broad recommendation for genetic marker motif type and repeat length can be made.

In our analysis of SSR loci in the genome of Daphnia pulex we describe both the catalogue of SSR sequences and several aspects of abundance and variation that are motif specific. Patterns that are motif specific include levels of heterozygosity, motif specific rates of repeat length variation, and motif specific patterns of NRUPs. Current models of simple sequence repeat evolution suggest that the abundance and variation at SSRs results from a balance of two opposing mutational forces. SSRs have a repeat number dependant rate of evolution resulting in the loss and gain of perfect repeat units. By contrast NRUPs disrupt repeat patterns and break down larger repeats into smaller ones. We also find a propensity for end nucleotide base substitution in SSRs, which was also reported in the chicken genome [18], suggesting that end nucleotide base substitution shapes the specific patterns of SSR abundance in multiple genomes. In addition to nucleotide end bias, we identify a significant difference in nucleotide starting preference for dimer and trimer classes.

\section{Methods}

\section{Sequence data}

The fasta sequences used in this study are from the Daphnia pulex genome project. The DOE Joint Genome Institute (JGI) and the Daphnia Genome Consortium (DGC) have sequenced 2,729,325 shotgun clones that result in $8.7 \times$ coverage of the Daphnia pulex genome. This sequence has been assembled using the JAZZ assembler, and consists of 9,080 scaffolds, containing a total of 1,591,853 reads, and 227.1 Mb. In this assembly, 103 scaffolds represent the N50. The sequences can be downloaded at JGI http://www.jgi.doe.gov/Daphnia/) and the DGC http://wfleabase.org/. The fasta sequences used in Figure 2 were taken from http://www.flybase.org (Drosophila melanogaster), http://www.wormbase.org (Caenorhabditis elegans), and http://www.yeastgenome. org (Saccharomyces cerevisiae).

\section{Detection of simple sequence repeats}

Programs written in PERL (available upon request) are used to count the number, length, location, and motif of all repeating motif size $1-100$ bps in the Daphnia n50 scaffolds, with a minimum repeat number of 3 repeats. A greedy algorithm is applied to finding the repeats. Once the first repeat is found, the location is noted and back matching of the repeat is used to determine the length of the repeated motif. The program allows for no mismatches (all repeats are perfect repeats). Only the smallest motif in the repeat is counted, larger nested motifs are counted as the lowest common repeating motif (eg. GAGAGAGA is counted as 4 repeats of GA, 
not one repeat of GAGAGAGA or two repeats of GAGA). Each motif is defined by the first occurrence of a repeating nucleotide (eg. GA and AG are unique motifs). Because this is a greedy algorithm, motifs of the largest size are identified, regardless of sequence identity (eg. AAAGAGAAAGAG is counted as two repeats of AAAGAG, not two homopolymeric runs of AAA).

\section{Measuring Heterozygosity}

To assay variation at SSR loci we used the AMOS reference assembler [32] to assemble the $8.7 \times$ sequences (average read length $=774$ ) to the JAZZ assembly at $90 \%$ identity to allow for an estimated $2-4 \%$ average sequence heterozygosity. This allows for heterozygous differences of between 6-8\% (46-62 nucleotides). In order to remove paralogy, coverage depth at each position was limited to a maximum of 16 and a minimum of 4 inclusive. Loci that met these criteria through the entire repeat were extracted from the AMOS output and analyzed for indels of perfect repeats and point mutations. Variants that are greater than 1 repeat unit in length were categorized as multi-step variations. In order to make a heterozygous call, we required a minimum of 2 consensus reads showing the variation. There were 966758 sites that only had one variant read for repeat differences and 1675976 sites that only had one variant read for SNP differences. The total number of reads covering these sites were 9468378 and 15642919 reads respectively, leading to a read error rate of $\sim 0.1$ for both types. Loci that had more than two alleles were thrown out of the analysis. There were 1220 loci that displayed two or more heterozygotes SNP calls, and 277 loci that displayed more than two or more heterozygous repeat calls. $(1497 / 6,062,268)$. The estimated frequency of paralogous loci in the dataset is $2.47 \mathrm{e}^{\wedge}-4$.

\begin{abstract}
Acknowledgements
The sequencing and portions of the analyses were performed the DOE Joint Genome Institute under the auspices of the U.S. Department of Energy's Office of Science, Biological and Environmental Research Program, and by the University of California, Lawrence Livermore National Laboratory under Contract No. W-7405-Eng-48, Lawrence Berkeley National Laboratory under Contract No. DE-AC02-05CH11231, Los Alamos National Laboratory under Contract No. W-7405-ENG-36 and in collaboration with the Daphnia Genomics Consortium (DGC) http://daphnia.cgb.indiana.edu. Additional analyses were performed by wFleaBase, developed at the Genome Informatics Lab of Indiana University with support to Don Gilbert from the National Science Foundation and the National Institutes of Health. Coordination infrastructure for the DGC is provided by The Center for Genomics and Bioinformatics at Indiana University, which is supported in part by the METACyt Initiative of Indiana University, funded in part through a major grant from the Lilly Endowment, Inc. Our work benefits from, and contributes to the Daphnia Genomics Consortium.
\end{abstract}

\section{Author details}

'Hubbard Center for Genome Studies, University of New Hampshire, Durham, NH 03824, USA. ${ }^{2}$ Department of Computer Science, University of New Hampshire, Durham, NH 03824, USA. ${ }^{3}$ Department of Biology, Indiana University, Bloomington, IN 47405, USA.

\section{Authors' contributions}

WS wrote the manuscript. AT, WS analyzed and interpreted data. AT, WS, RDB, WKT contributed to conception and design. ML and WKT participated in critical manuscript revision. All authors read and approved the final manuscript.

Received: 24 May 2010 Accepted: 3 December 2010

Published: 3 December 2010

\section{References}

1. Kashi Y, King D: Simple sequence repeats as advantageous mutators in evolution. Trends in Genetics 2006, 22(5):253-259.

2. Denver D, Morris K, Kewalramani A, Harris K, Chow A, Estes S, Lynch M, Thomas WK: Abundance, Distribution, and Mutation Rates of Homopolymeric Nucleotide Runs in the Genome of Caenorhabditis elegans. J Mol Evol 2004, 58:584-595.

3. Lynch M, Sung W, Morris K, Crown N, Landry C, Dopman E, Dickinson WJ, Okamoto K, Kulkarni S, Thomas WK: A Genome-wide View of the Spectrum of Spontaneous Mutations in Yeast. Proc Natl Acad Sci USA 105(27):9272-7.

4. Toth G, Gaspari Z, Jurka J: Microsatellites in different eukaryotic genomes: survey and analysis. Genome Res 2000, 10:967-981.

5. Kruglyak S, Durett R, Schug M, Aquadro CF: Distribution and abundance of microsatellites in the yeast genome can be explained by a balance between slippage events and point mutations. Mol Biol Evol 2000, 17:1210-1219.

6. Denver D, Feinberg S, Estes S, Thomas WK, Lynch M: Mutation Rates, Spectra and Hotspots in Mismatch Repair-Deficient Caenorhabditis elegans. Genetics 2005, 170:108-113.

7. Sia EA, Kokoska R, Dominska M, Greenwell P, Petes T: Microsatellite Instability in Yeast: Dependence on Repeat Unit Size and DNA Mismatch Repair Genes. Mol Cell Bio 1997, 17(5):2851-2858.

8. Hawk J, Stefanovic L, Boyer J, Petes T, Farber R: Variation in efficiency of DNA mismatch repair at different sites in the yeast genome. Proc Natl Acad Sci USA 2005, 102:8639-8643.

9. Eisen J: A phylogenomic study of the MutS family of proteins. Nucleic Acids Res 1998, 26(18):4291-4300.

10. Gragg H, Harfe B, Robertson SJ: Base composition of mononucleotide runs affects DNA polymerase slippage and removal of frameshift intermediates by mismatch repair in Saccharomyces cerevisiae. Mol Cell Bio 2002, 22(24):8756-8762.

11. Schlötterer C: Evolutionary dynamics of microsatellite DNA. Chromosoma 2000, 109:365-371.

12. Bachtrog D, Agis M, Imhof M, Schlötterer C: Microsatellite Variability Differs Between Dinucleotide Repeat Motifs-Evidence from Drosophila melanogaster. Mol Biol Evol 2000, 17(9):1227-1285.

13. wFleaBase. [http://wFleaBase.org/].

14. Zhou Y, Bizzaro J, Marx K: Homopolymer tract lengths dependent enrichments in functional regions of 27 eukaryotes and their novel dependence on the organism DNA $(\mathrm{G}+\mathrm{C}) \%$ composition. BMC Genomics 2004, 5:95.

15. JGI Genome Portal. [http://www.jgi.doe.gov/Daphnia/].

16. Nadir E, Margalit $H$, Gallily T, Bensasson SA: Microsatellite spreading in the human genome: Evolutionary mechanisms and structural implications. Proceedings of the National Academy of Sciences 1996, 93:6470-6475.

17. Kelkar YD, Tyekucheva S, Chiaromonte F, Makova KD: The genome-wide determinants of human and chimpanzee microsatellite evolution. Genome Res 2008, 18:30-38.

18. Brandstrom $\mathrm{M}$, Ellegren $\mathrm{H}$ : Genome-wide analysis of microsatellite polymorphism in chicken cirvumventing the ascertainment bias. Genome research 2008

19. Ohta T, Kimura M: A model of mutation appropriate to estimate the number of electrophoretically detectable alleles in a finite population. Genet Res 1973, 22:201-204.

20. Kruglyak S, Durett R, Schug M, Aquadro CF: Equilibrium distributions of microsatellite repeat length resulting from a balance between slippage events and point mutations. Proc Natl Acad Sci USA 1998, 95:10774-10778.

21. Lai $Y$, Sun F: The relationship between microsatellite slippage mutation rate and the number of repeat units. Mol Biol Evol 20:2123-2131.

22. Ellegren $\mathrm{H}$ : Heterogeneous mutation processes in human microsatellite sequences. Nature Genetics 2000, 24:401-402. 
23. Rose O, Falush D: A Threshold Size for Microsatellite Expansion. Mol Biol Evol 15(5):613-615.

24. Ellegren H: Microsatellites: Simple sequences with complex evolution. Nature Review Genetics 2004, 5:435-444.

25. Xu X, Peng M, Fang Z: The direction of microsatellite mutations is dependent upon allele length. Nature Genet 2000, 24:396-399.

26. Seyfert A, Cristescu M, Frisse L, Schaack S, Thomas WK, Lynch M: The rate and spectrum of microsatellite mutation in Caenorhabditis elegans and Daphnia pulex. Genetics 2008, 178:2113-2121.

27. Schug M, Hutter C, Noor M, Aquadro C: Mutation and evolution of microsatellites in Drosophila melanogaster. Genetica 1998, 102(103):359-367.

28. Bagshaw A, Pitt J, Gemmell N: High frequency of microsatellites in S. cerevisiae meiotic recombination hotspots. BMC Genomics 2008, 9:49.

29. Cristescu M, Colbourne J, Radivojac J, Lynch M: A microsatellite-based genetic linkage map of the waterflea, Daphnia pulex: On the prospect of crustacean genomics. Genomics 2006, 88:415-430.

30. Payseur B, Nachman M: Microsatellite Variation and Recombination Rate in the Human Genome. Genetics 2000, 156:1285-1298.

31. Birdsell J: Integrating genomics, Bioinformatics, and Classical Genetics to Study the Effects of Recombination on Genome Evolution. Mol Bio Evol 19(7):1181-1197.

32. AMOS: A Modular Open-Source Assembler [http://amos.sourceforge.net/].

doi:10.1186/1471-2164-11-691

Cite this article as: Sung et al: Simple sequence repeat variation in the Daphnia pulex genome. BMC Genomics 2010 11:691.

\section{Submit your next manuscript to BioMed Central} and take full advantage of:

- Convenient online submission

- Thorough peer review

- No space constraints or color figure charges

- Immediate publication on acceptance

- Inclusion in PubMed, CAS, Scopus and Google Scholar

- Research which is freely available for redistribution

Submit your manuscript at www.biomedcentral.com/submit
Biomed Central 